\title{
ThFeAsN in Relation to Other Iron-Based Superconductors
}

\author{
David J. Singh \\ ${ }^{a}$ Department of Physics and Astronomy, University of Missouri, Columbia, MO \\ 65211-7010 USA
}

\begin{abstract}
The electronic structure, magnetic and structural properties of the superconductor ThFeAsN are discussed in relation to the Fe-pnictide and Fe-chalcogenide superconductors based on results of first principles calculations. The electronic structure is that of a high density of states semimetal. It shows approximately nested hole and electron Fermi surfaces of $\mathrm{Fe} d$ character involving the $x z, y z$ and $x y$ orbitals. There is a strong tendency towards magnetism at the GGA level, and this magnetism is important for describing the Fe-As bonding, but greatly overestimates the magnetic ordering. The lowest energy magnetic state at the GGA level is the stripe order, and this particular order couples strongly to electrons near $E_{F}$. ThFeAsN is therefore strongly similar to the Fe-pnictide family of superconductors, although it is a particularly anisotropic member.
\end{abstract}

\section{Introduction}

Superconductivity at $T_{c}=30 \mathrm{~K}$ was recently reported in the tetragonal compound ThFeAsN, without doping. [1] The compound does not show antiferromagnetic ordering, and occurs in the same crystal structure as LaFeAsO and $\mathrm{LaFePO}$. However, LaFeAsO, while tetragonal at high temperature has a structural distortion to an orthorhombic symmetry below $\sim 160 \mathrm{~K}$ and is antiferromagnetic below $\sim 145 \mathrm{~K}$. Moreover, it is not superconducting without doping or pressure. [2, 3] LaFePO on the other hand stays tetragonal but only becomes superconducting at $\sim 4 \mathrm{~K}$, [4] i.e. much lower than electron doped $\mathrm{LaFeAsO}$, which has $T_{c} \sim 26 \mathrm{~K}$. Substitution of the smaller rare earth ions, e.g. Ce, Nd, Sm and $\mathrm{Pr}$ for La in electron doped $\operatorname{LaFeAs}(\mathrm{O}, \mathrm{F})$ leads to increases in $T_{c}$ to over 56 K. [5] Similar to LaFeAsO, the parent compounds are antiferromagnetic and non-superconducting at ambient pressure.

From a structural point of view, ThFeAsN has a significantly shorter $c$-axis than $\mathrm{LaFeAsO}(8.526 \AA$ vs. $8.737 \AA)$, but has a larger $a$-axis $(4.037 \AA$ vs. $4.030 \AA$ ). This means that the Fe-As planes, presumably responsible for superconductivity, have a larger in-plane spacing, opposite to the effect of pressure

Email address: singhdj@missouri.edu (David J. Singh) 
on LaFeAsO and also opposite to the effect of substituting smaller rare earth ions. The shorter $c$-axis might suggest a more three dimensional electronic structure, but this would depend on the details of the hopping through ThN vs. LaO. One may also note that the behavior of the compound, in particular superconductivity without doping, is reminiscent of some of the Fe-chalcogenide superconductors, such as FeSe. [6, 7, 8] The purpose of this work is to examine the electronic and related properties of ThFeAsN using density functional calculations.

\section{Approach}

The present calculations were done using standard density functional calculations with the PBE generalized gradient approximation (PBE-GGA), [9] and the general potential linearized augmented planewave (LAPW) method [10] as implemented in the WIEN2k code. [11] The LAPW sphere radii were $2.4 \mathrm{bohr}$, 2.2 bohr, 2.2 bohr and 1.9 bohr for Th, Fe, As and N, respectively. Converged basis sets consisting of LAPW functions up to a cut-off of $R_{\min } k_{\max }=8$ $\left(R_{\min }=1.9 \mathrm{bohr}\right.$, the $\mathrm{N}$ radius) plus local orbitals were used. The results shown were obtained in a scalar relativistic approximation, but tests were performed including spin-orbit. We found only very small effects due to spin-orbit, in spite of the fact that the compound contains the actinide element Th. The reason is that the $\mathrm{Th}$ is very electropositive relative to $\mathrm{N}$, and as a result the Th contributions to the occupied valence states are very small (see below).

\section{Results and Discussion}

We begin with the structure and magnetism. ThFeAsN is not reported to show magnetic ordering. [1] Calculations using the experimental crystal structure without magnetism predict large forces on the As atoms. Relaxing the atomic positions in the unit cell gives large changes in the As position. Specifically, the As height above the Fe plane changes from $1.305 \AA$ to $1.195 \AA$, leading to a decrease in Fe-As bond length of $0.058 \AA$. This is far outside any reasonable error in the experimental structure. If magnetism is included an antiferromagnetic stripe state like that of undoped $\mathrm{LaFeAsO}$ is obtained. The calculated moment in the Fe LAPW sphere is $2.01 \mu_{B}$, and in this case an As position of $1.274 \AA$ above the Fe plane is obtained, in much better accord with experiment. This conundrum in which standard GGA calculations yield excessive Fe-As bonding in disagreement with experimental crystal structures without magnetism, and better structures at the expense of greatly overestimated magnetic moments when magnetism is included is a characteristic of the Fe-based superconductors. [12] It has been associated with the unusual spin-fluctuations in them, [13] although the subtle nature of the spin fluctuations in the Fe-based superconductors remains to be fully understood. $[5,14,15,16]$

We did magnetic calculations for three ordering patterns: ferromagnetic, nearest neighbor checkerboard antiferromagnetic and stripe antiferromagnetic. 


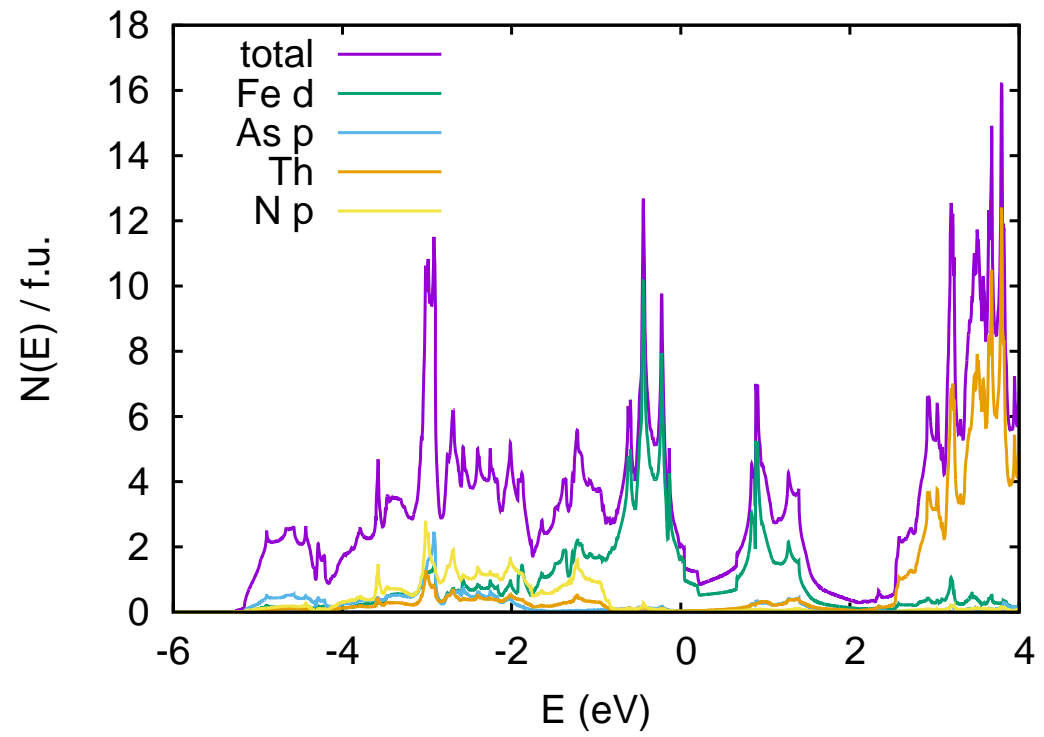

Figure 1: Calculated electronic density of states of ThFeAsN and projections on a per formula unit basis. The Fermi energy is at $0 \mathrm{eV}$. 

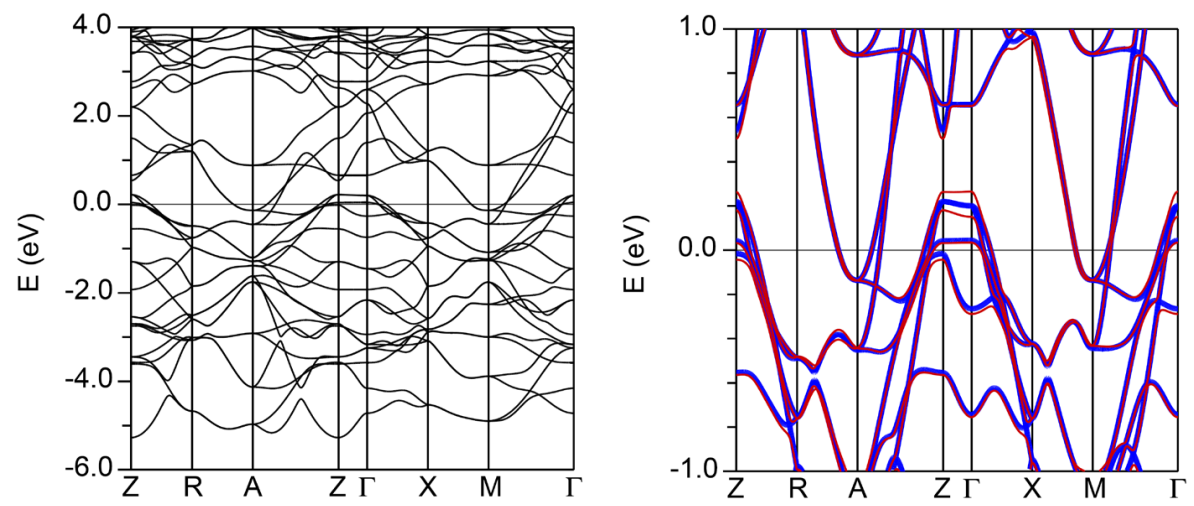

Figure 2: Scalar relativistic band structure (left) and a blow up near $E_{F}$ showing the scalar relativistic bands as heavy blue lines and the bands with spin-orbit as lighter red lines (right). The Fermi energy is at $0 \mathrm{eV}$.

As mentioned the lowest energy is for the stripe pattern. With the experimental crystal structure and on a per formula unit basis, the ferromagnetic order has an Fe moment defined by the spin polarization in the Fe LAPW sphere of 0.36 $\mu_{B}$ and energy $-0.002 \mathrm{eV}$ relative to the non-spin-polarized case. The nearest neighbor checkerboard order has moment $1.81 \mu_{B}$ and energy $-0.059 \mathrm{eV}$, while the stripe order has moment $2.01 \mu_{B}$ and energy $-0.151 \mathrm{eV}$.

The calculated energy scales for ordering and moment formation are similar to each other. The difference in energy of the strip order and the ferromagnetic order and that between the lowest energy magnetic and the non-spinpolarized case are nearly the same. Additionally, moment size depends strongly on the particular order. This behavior is similar to the behavior of other Febased superconducting systems in density functional calculations. It also may be taken as indicating itinerant character of the magnetism, [17, 18, 19] although we note that this characterization has been a subject of controversy in Fe-superconductors. $[15,20]$ In any case, the results show a similar behavior of ThFeAsN to the other Fe-based superconductors.

We now turn to the electronic structure. The calculated electronic density of states (DOS) is shown in Fig. 1. The Fermi energy, $E_{F}$, is located on the low energy side of a dip in the DOS, similar to LaFeAsO and most other Febased superconductors. [17] This dip at an electron count of six per Fe does not correspond to a tetrahedral crystal field (in which the 4-fold $e_{g}$ manifold would be lower) but instead reflects metal-metal bonding in addition to the interaction of $\mathrm{Fe}$ with the As ligands, as was discussed previously for other Fe-based superconductors. [17, 21]

The value of the DOS at $E_{F}$ is $N\left(E_{F}\right)=2.02 \mathrm{eV}^{-1}$ per formula unit and as seen in the projection is more than $80 \%$ from Fe $d$ states. This places the compound at a Stoner instability. [22, 23] This is the reason for the marginal ferromagnetic instability, mentioned above. The calculated bare specific heat 
coefficient from the density of states is $\gamma_{b a r e}=4.8 \mathrm{~mJ} / \mathrm{mol} \mathrm{K}^{2}$ on a per formula unit basis. Note, however, that in analogy with other Fe-based superconductors there may be a substantial enhancement due to spin-fluctuations or other correlations. [24, 25, 26]

The effect of spin orbit on the density of states is small. $N\left(E_{F}\right)$ with spin orbit is $2.12 \mathrm{eV}^{-1}$. The Th states are unoccupied, while the As and $\mathrm{N} p$ are below $E_{F}$. The fact that the Th states are removed from the Fermi level explains the relative insensitivity of the calculation to spin-orbit. It also means that the Th$\mathrm{N}$ layers can be regarded as $(\mathrm{ThN})^{+1}$ units that serve to stabilize the structure and donate one electron to $(\mathrm{FeAs})^{-1}$ metallic sheets, similar to LaFeAsO. Also because there is almost no Th character at $E_{F}$, the ionic $(\mathrm{ThN})^{+1}$ sheets are expected to be insulating, which leads to a rather two dimensional electronic structure for the compound, as discussed below.

The carriers at $E_{F}$ are strongly coupled to the magnetism, particularly for the stripe ordering. We obtain a strongly reduced, $N\left(E_{F}\right)=0.31 \mathrm{eV}^{-1}$ per formula unit for this case. This is also a characteristic of the Fe-based superconductors. [27, 28] The nearest neighbor checkerboard antiferromagnetic state does not gap the DOS in this way. This order gives $N\left(E_{F}\right)=4.69^{-1}$ per formula unit, i.e. higher than the non-magnetic state. This shows that similar to other Fe-based superconductors, [20] the magnetism involves Fe $d$ states both near and away from $E_{F}$ (i.e. the whole $d$ band), although the particular ordering pattern realized is strongly influenced by the low energy band structure.

The calculated band structure is shown in Fig. 2, including a blow up of the low energy region comparing calculations with and without spin-orbit. Plots emphasizing the different Fe $d$ orbital characters are given in Fig. 3. The largest evident effect of spin-orbit is a splitting of the $x z, y z$ derived bands above $E_{F}$ that make up the two main hole Fermi surfaces along the $\Gamma-Z$ direction. The effect is, however, small at $E_{F}$.

There are five bands crossing $E_{F}$ : three hole bands, two of which are of $x z, y z$ character, and one of which is of $x y$ character and two electron bands of predominantly $x z, y z$ character hybridized with $x y$ character. The resulting Fermi surfaces are shown in Fig. 4. There are three very two dimensional and nearly circular hole cylinders around the zone center and two corrugated electron cylinders at the zone corner. In addition, it is notable that there is a $z^{2}$ band with a maximum just below $E_{F}$ at $Z$. This band differs from other bands crossing $E_{F}$ in that it has considerably more dispersion in the $k_{z}$ direction (see the $\Gamma$ - $Z$ line in Fig. 2).

The electron cylinders can be regarded as being derived from the intersection of two cylinders of elliptical cross-section with the outer lobes having more $x y$ character and the narrower (inner) part having $x z, y z$ character. This is similar to the other Fe-based superconductors, including FeSe. [29, 17, 30, 31, 32, 33] In particular, ThFeAsN is a high density of states, low carrier density semimetal, with electron and hole sections separated by $\left(\frac{\pi}{a}, \frac{\pi}{a}\right)$ and having similar orbital character. This aspect of the electronic structure plays a central role in the magnetism of Fe-based superconductors, in particular the tendency for a stripe magnetic order that couples strongly to the carriers at $E_{F}$, and the sign changing 

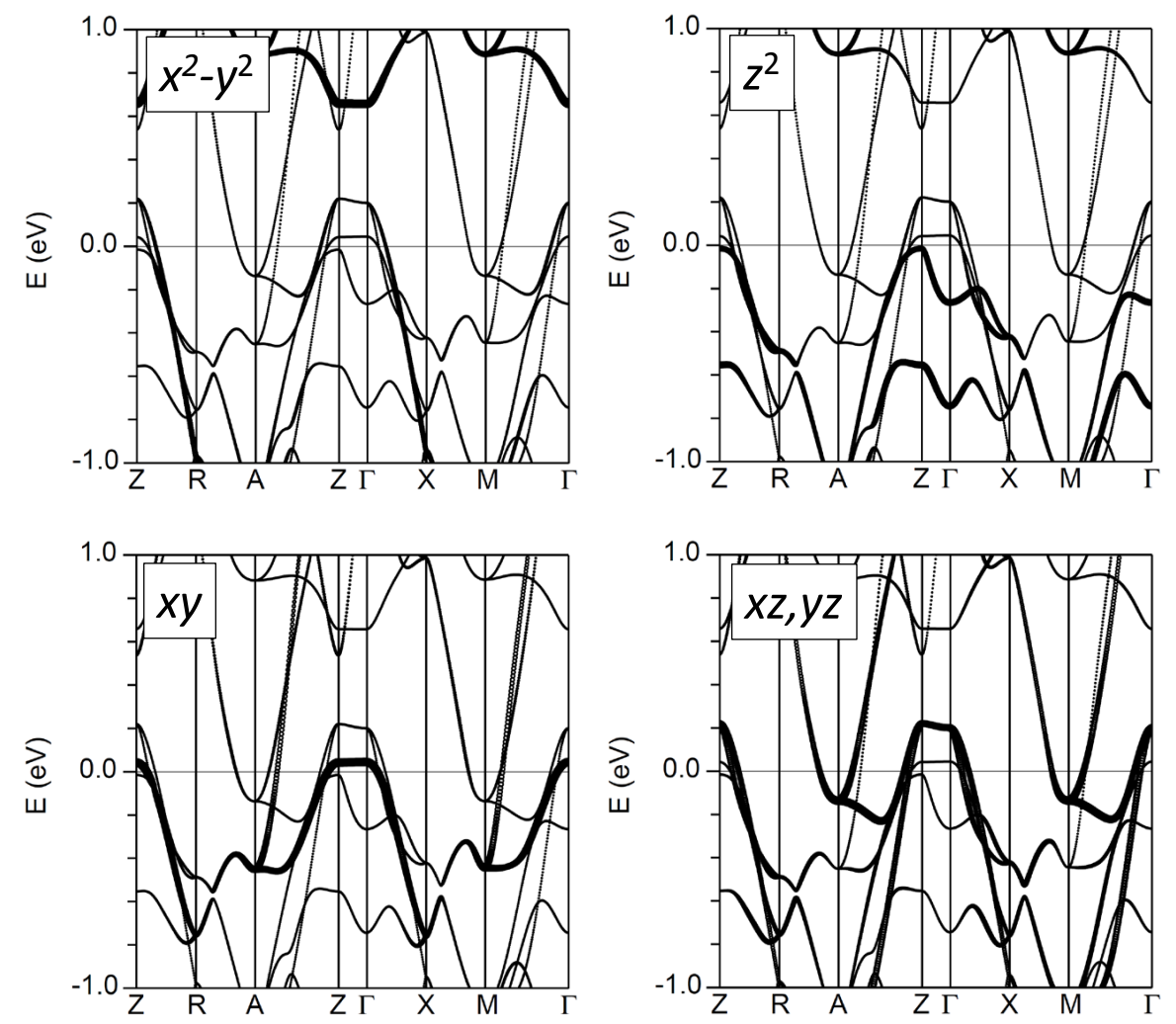

Figure 3: Band structure plots emphasizing via "fat bands" the different Fe $d$ orbital characters. The coordinates for defining the Fe $d$ orbitals are such that $z$ is along the $c$-axis, while the $x$ and $y$ directions are on the Fe-Fe bonds (i.e. rotated $45^{\circ}$ with respect to the two $\mathrm{Fe}$ atom unit cell $a$ and $b$ axes). 


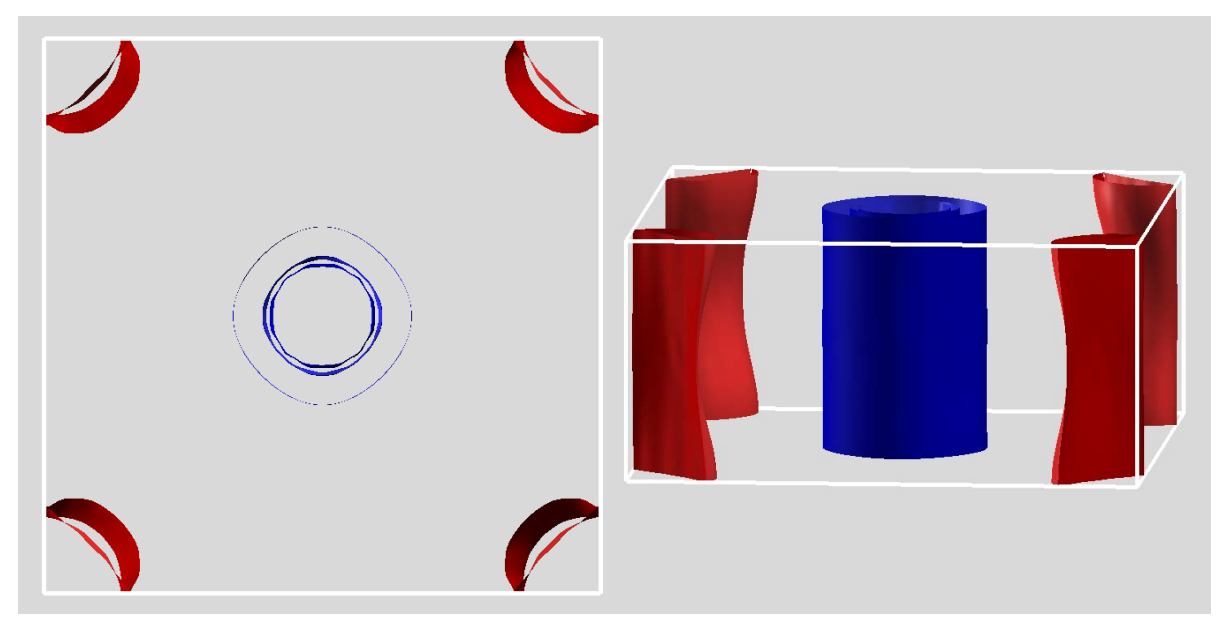

Figure 4: Calculated Fermi surfaces of ThFeAsN. Hole sheets are shown as blue and electron sheets as red.

$s$-wave superconducting state that is proposed to occur in these compounds. $[18,19,34]$

The hole surfaces contain $0.051,0.068$ and 0.153 holes per unit cell on a both spins basis, while the electron surfaces contain 0.109 and 0.163 electrons per cell also counting both spins. The calculated plasma frequencies are $\Omega_{p, x x}=\Omega_{p, z z}=2.31 \mathrm{eV}$ and $\Omega_{p, z z}=0.36 \mathrm{eV}$.

In the constant scattering time approximation, the conductivity, $\sigma \propto \Omega_{p}^{2}$. The predicted conductivity anisotropy based on this is high, $\sigma_{x x} / \sigma_{z z} \sim 40$. Structural changes, e.g. by alloying, that bring the $z^{2}$ hole band to $E_{F}$ will reduce this anisotropy. In any case, this places ThFeAsN among the more anisotropic of the Fe-based superconductors particularly when compared to e.g. the $\mathrm{ThCr}_{2} \mathrm{Si}_{2}$ structure materials, such as $\mathrm{BaFe}_{2} \mathrm{As}_{2}$. [35, 36] This is a consequence of the fact that the $(\mathrm{ThN})^{+}$sheets, although thinner than the $(\mathrm{LaO})^{+}$sheets of $\mathrm{LaFeAsO}$, are highly insulating.

\section{Summary and Conclusions}

To summarize, ThFeAsN shows several differences from the other Fe-As superconductors, most notably the superconductivity and lack of magnetic ordering in the absence of doping, but nonetheless, from an electronic structure point of view it is very similar to the other Fe-based superconductors. The electronic structure is that of a high density of states semimetal. It shows approximately nested hole and electron Fermi surfaces of Fe $d$ character involving the $x z, y z$ and $x y$ orbitals. There is a strong tendency towards magnetism at the GGA level, and this magnetism is important for describing the Fe-As bonding, but greatly overestimates the magnetic ordering. The lowest energy magnetic state 
at the GGA level is the stripe order, and this particular order couples strongly

to electrons near $E_{F}$.

\section{Acknowledgements}

Support from the Department of Energy through the MAGICS center is gratefully acknowledged.

\section{References}

[1] C. Wang, Z. C. Wang, Y. M. Mei, Y. K. Li, L. Li, Z. T. Tang, Y. Liu, P. Zhang, H. F. Zhai, Z. A. Xu, G. H. Cao, A new ZrCuSiAs-type superconductor: ThFeAsN, J. Am. Chem. Soc. 138 (2016) 2170-2173.

[2] Y. Kamihara, T. Watanabe, M. Hirano, H. Hosono, J. Am. Chem. Soc. 130 (2008) 3296.

[3] C. de la Cruz, Q. Huang, J. W. Lynn, J. Li, W. Ratcliff II, J. L. Zaretsky, H. A. Mook, G. F. Chen, J. L. Luo, N. L. Wang, P. Dai, Nature (London) $453(2008) 899$.

[4] Y. Kamihara, H. Hiramatsu, M. Hirano, R. Kawamura, H. Yanagi, T. Kamiya, H. Hosono, J. Am. Chem. Soc. 128 (2006) 10012.

[5] D. C. Johnston, The puzzle of high temperature superconductivity in layered iron pnictides and chalcogenides, Adv. Phys. 59 (2010) 803.

[6] F. C. Hsu, J. Y. Luo, K. W. Yeh, T. K. Chen, T. W. Huang, P. M. Wu, Y. C. Lee, Y. L. Huang, Y. Y. Chu, D. C. Yan, M. K. Wu, Proc. Nat. Acad. Sci. (USA) 105 (2008) 14262.

[7] Y. Mizuguchi, F. Tomioka, S. Tsuda, T. Yamaguchi, Y. Takano, Appl. Phys. Lett. 93 (2008) 152505.

[8] D. J. Singh, Sci. Tech. Adv. Mater. 13 (2012) 054304.

[9] J. P. Perdew, K. Burke, M. Ernzerhof, Generalized gradient approximation made simple, Phys. Rev. Lett. 77 (18) (1996) 3865-3868.

[10] D. J. Singh, L. Nordstrom, Planewaves Pseudopotentials and the LAPW Method, 2nd Edition, Springer, Berlin, 2006.

[11] P. Blaha, K. Schwarz, G. Madsen, D. Kvasnicka, J. Luitz, WIEN2k, An Augmented Plane Wave + Local Orbitals Program for Calculating Crystal Properties, K. Schwarz, Tech. Univ. Wien, Austria, 2001.

[12] I. I. Mazin, M. D. Johannes, L. Boeri, K. Koepernik, D. J. Singh, Phys. Rev. B 78 (2008) 085104. 
[13] F. Bondino, E. Magnano, M. Malvestuto, F. Parmigiani, M. A. McGuire, A. S. Sefat, B. C. Sales, R. Jin, D. Mandrus, E. W. Plummer, D. J. Singh, N. Mannella, Phys. Rev. Lett. 101 (2008) 267001.

[14] J. Paglione, R. L. Greene, Nature Physics 6 (2010) 645.

[15] C. Wang, R. Zhang, F. Wang, H. Luo, L. P. Regnault, P. Dai, Y. Li, Phys. Rev. X 3 (2013) 041036.

[16] H. Hosono, K. Tanabe, E. Takayama-Muromachi, H. Kageyama, S. Yamanaka, H. Kumakura, M. Nohara, H. Hiramatsu, S. Fujitsu, Sci. Tech. Adv. Mater. 16 (2015) 033503.

[17] D. J. Singh, M. H. Du, Phys. Rev. Lett. 100 (2008) 237003.

[18] I. I. Mazin, D. J. Singh, M. D. Johannes, M. H. Du, Phys. Rev. Lett. 101 (2008) 057003.

[19] K. Kuroki, S. Onari, R. Arita, H. Usui, Y. Tanaka, H. Kontani, H. Aoki, Phys. Rev. Lett. 102 (2009) 109902.

[20] M. D. Johannes, I. I. Mazin, Phys. Rev. B 79 (2009) 220510.

[21] D. J. Singh, Physica 469 (2009) 418.

[22] E. C. Stoner, Collective electron ferromagnetism II: energy and specific heat, Proc. R. Soc. London Ser. A 169 (1939) 339-371.

[23] J. F. Janak, Uniform susceptibilities of metallic elements, Phys. Rev. B 16 (1977) 255-262.

[24] M. Yi, D. H. Lu, J. G. Analytis, J.-H. Chu, S.-K. Mo, R.-H. He, R. G. Moore, X. J. Zhou, G. F. Chen, J. L. Luo, N. L. Wang, Z. Hussain, D. J. Singh, I. R. Fisher, Z.-X. Shen, Phys. Rev. B 80 (2009) 024515.

[25] M. M. Qazilbash, J. J. Hamlin, R. E. Baumbach, L. Zhang, D. J. Singh, M. B. Maple, D. N. Basov, Nature Physics 5 (2009) 647.

[26] A. Tamai, A. Y. Ganin, E. Rozbicki, J. Bacsa, W. Meevasana, P. D. C. King, M. Caffio, R. Schaub, S. Margadonna, K. Prassides, M. J. Rosseinsky, F. Baumberger, Phys. Rev. Lett. 104 (2010) 097002.

[27] M. Yi, D. H. Lu, J. G. Analytis, J.-H. Chu, S.-K. Mo, R.-H. He, M. Hashimoto, R. G. Moore, I. I. Mazin, D. J. Singh, Z. Hussain, I. R. Fisher, Z.-X. Shen, Phys. Rev. B 80 (2009) 174510.

[28] S. E. Sebastian, J. Gillett, N. Harrison, P. H. C. Lau, D. J. Singh, C. H. Mielke, G. G. Lonzarich, Quantum oscillations in the parent magnetic phase of an iron arsenide high temperature superconductor, Journal of Physics: Condensed Matter 20 (42) (2008) 422203. 
[29] S. Lebegue, Phys. Rev. B 75 (2007) 035110.

[30] S. Raghu, X. L. Qi, C. X. Liu, D. J. Scalapino, S. C. Zhang, Phys. Rev. B 77 (2008) 220503.

[31] M. J. Calderon, B. Valenzuela, E. Bascones, Phys. Rev. B 80 (2009) 094531.

[32] A. Subedi, L. Zhang, D. J. Singh, M. H. Du, Phys. Rev. B 78 (2008) 134514.

[33] M. M. Korshunov, I. Eremin, Phys. Rev. B 78 (2008) 140509.

[34] A. D. Christianson, E. A. Goremychkin, R. Osborn, S. Rosenkranz, M. D. Lumsden, C. D. Malliakis, I. S. Todorov, H. Claus, D. Y. Chung, M. G. Kanatzidis, R. I. Bewley, T. Guidi, Nature (London) 456 (2008) 930.

[35] H. Q. Yuan, J. Singleton, F. F. Balakirev, S. A. Baily, G. F. Chen, J. L. Luo, N. L. Wang, Nature (London) 457 (2009) 565.

[36] P. Vilmercati, A. Fedorov, I. Vobornik, U. Manju, G. Panaccione, A. Goldoni, A. S. S. an M A McGuire, B. C. Sales, R. Jin, D. Mandrus, D. J. Singh, N. Mannella, Phys. Rev. B 79 (2009) 220503. 